\title{
ON THE COMPLEX DIMENSIONS OF NONLATTICE FRACTAL STRINGS IN CONNECTION WITH DIRICHLET POLYNOMIALS
}

\author{
E. DUBON AND J.M. SEPULCRE
}

\begin{abstract}
In this paper we give a new characterization of the closure of the set of the real parts of the zeros of a particular class of Dirichlet polynomials which is associated to the set of dimensions of fractality of certain fractal strings. We show, for some representative cases of nonlattice Dirichlet polynomials, that the real parts of their zeros are dense in their associated critical intervals, confirming the conjecture and the numerical experiments made by M. Lapidus and M. van Frankenhuysen in several papers.
\end{abstract}

\section{INTRODUCTION}

An expression of the form

$$
m_{0} r_{0}^{z}+m_{1} r_{1}^{z}+\ldots+m_{n} r_{n}^{z},
$$

with $r_{0}>r_{1}>\ldots>r_{n}>0$ (called scaling ratios) and multiplicities $m_{j} \in \mathbb{C}$, $j=0,1, \ldots, n$, is called a Dirichlet polynomial. Without loss of generality, for purposes of zeros, we assume the normalization

$$
f(z)=1+\sum_{j=1}^{n} m_{j} r_{j}^{z}=1+\sum_{j=1}^{n} m_{j} e^{-z w_{j}}
$$

where $w_{j}=-\log r_{j}$ for $j=1, \ldots, n$ (called weights). If some ratio $\frac{\log r_{j}}{\log r_{1}}, j \geq 2$, is irrational, they are called nonlattice Dirichlet polynomials. On the contrary, when $r_{j}=r^{k_{j}}$ are integral powers of a common base $r$, they are called lattice Dirichlet polynomials.

The zeros of the Dirichlet polynomials (1.1) are connected to the concept of fractal string $[4,5,6]$ : a set that is a disjoint union of open intervals whose lengths form a sequence $\mathcal{L}=l_{1}, l_{2}, \ldots$ of finite total length $\sum_{j=1}^{\infty} l_{j}$. Important information on the geometry of $\mathcal{L}$ is contained in its geometric zeta function, defined by

$$
\zeta_{\mathcal{L}}(z):=\sum_{j=1}^{\infty} l_{j}^{z}
$$

Date: November 14, 2013.

2010 Mathematics Subject Classification. 30Axx, 28A80, 11M41.

Key words and phrases. Dirichlet Polynomials; Fractal Strings; Complex Dimensions of Fractal Strings; Set of dimensions of fractality; Zeros of entire functions.

Accepted Manuscript in Experimental Mathematics.

The second author is the corresponding author and he was partially supported by Vicerrectorado de Investigación, Desarrollo e Innovación de la Universidad de Alicante under project GRE11-23. 
If $\sigma$ denotes the value inf $\left\{\alpha \in \mathbb{R}: \sum_{j=1}^{\infty} l_{j}^{\alpha}<\infty\right\}$, then $\{z \in \mathbb{C}: \operatorname{Re} z>\sigma\}$ is the largest open half-plane on which the series (1.2) converges. Clearly, $0 \leq \sigma \leq 1$.

The poles of the meromorphic extension of $\zeta_{\mathcal{L}}$ are called complex dimensions of the fractal string $\mathcal{L}$ and the set of dimensions of fractality of a fractal string is defined as the closure of the set of real parts of its complex dimensions.

It is worthwhile to remark that, in the theory of self-similar strings, the geometric importance of the complex dimensions is justified because they appear in the explicit formula for the volume $V(\epsilon)$ of the inner tubular neighborhood of the boundary of $\mathcal{L}$ $[5$, p.42]. Moreover, the properties of the set of dimensions of fractality are related to the concept of the oscillation and, more precisely, the real parts of the complex dimensions determine the amplitude of these oscillations [5, Remark 8.2].

For the case of self-similar strings $\mathcal{L}$ with scaling ratios $r_{1}, r_{2}, \ldots, r_{N}$ and gaps $g_{1}, \ldots, g_{K}$ (whose construction is reminiscent of the construction of the Cantor set), the meromorphic continuation to the whole complex plane of $\zeta_{\mathcal{L}}$ has the form

$$
\zeta_{\mathcal{L}}(z)=\frac{L^{z} \sum_{k=1}^{K} g_{k}^{z}}{1-\sum_{j=1}^{N} r_{j}^{z}}
$$

where $L$ is the total length of $\mathcal{L}[5$, Theorem 5.2]. Thus the complex dimensions of $\mathcal{L}$ are the zeros of the Dirichlet polynomial $P(z)=1-\sum_{j=1}^{N} r_{j}^{z}$ and its set of dimensions of fractality is given by $R_{P}:=\overline{\{\operatorname{Re} z: P(z)=0\}}$.

In the lattice case, the complex roots are obtained by finding the complex solutions of a polynomial equation and, in that case, the complex roots lie periodically on finitely many vertical lines [6, Theorem 2.16], such as occurs for example with the Cantor and Fibonacci strings [5, Example 2.2.1 and Example 2.2.2].

The recent paper [10] is focused on the Dirichlet polynomials $f(z)$ which are of the form (1.1) with weights that are linearly independent over the rationals and, hence, uniquely connected to a determined class of nonlattice fractal strings, which form the generic nonlattice self-similar case. In that paper, it was proved that the set of dimensions of fractality associated to these strings is either the entire interval that contains the projection of their complex dimensions or the union of at most $n$ disjoint non-degenerate closed intervals.

However, in this paper the interest is mainly focused on the Dirichlet polynomials

$$
L_{n}(z):=1-2^{z}-\ldots-n^{z}=1-e^{z \log 2}-\ldots-e^{z \log n},
$$

for integer values of $n \geq 2$, which are the prototype of the nonlattice case (nongeneric). Thus, the results of [10] cannot be applied, except for the case $n=3$, to the functions $L_{n}(z)$ because they have some distinct weights which are linearly dependent over the rationals (for example: $\log 2$ and $\log 4$ ). Therefore, the techniques used in this paper in order to find a new characterization of the set of points of the set $R_{L_{n}}:=\overline{\left\{\operatorname{Re} z: L_{n}(z)=0\right\}}$, for the general case, are rather different.

Notice that, for the case $n=3, L_{3}(-z)$ is the function directly obtained by considering the Two-Three string $\mathcal{L}$, with scaling factors $\frac{1}{2}$ and $\frac{1}{3}$, a simple gap and total length equal to $6[6, \mathrm{p} .49]$. The case $n=4$ is studied in $[6, \mathrm{p} .70,1.12]$ or $[5$, section 2.2.4].

Hence this paper arises from the need to obtain new theorems of density of real parts of the zeros for the case that the set of weights of the Dirichlet polynomials is 
not necessarily linearly independent over the rationals (or equivalently, the scaling ratios generate a multiplicative group of rank $>1$ but not of maximal rank) and, in this way, to connect these density properties with the underlying self-similar fractal strings.

The main results of this paper can be summarized as follows:

i) In Section 2, we will give a method in order to compute the minimum and an upper bound for the maximum of the critical interval where the real parts of the zeros of the nonlattice Dirichlet polynomials $L_{n}(z)$, for $n \geq 2$, are located.

ii) For these functions $L_{n}(z)$, we will prove in Section 3 that $R_{L_{n}}$ entirely coincides with its maximum interval of density for the cases $n=2,3,4$ and we will show a large set contained in $R_{L_{5}}$.

iii) We will establish in Section 4 a new characterization of the set of points of $R_{L_{n}}, n \geq 2$, through the level curves of an auxiliary function, which leads us to obtain some points that are in $R_{L_{n}}$.

iv) In Section 5, we will calculate the set of dimensions of fractality of other known fractal strings such as the golden or the golden+ string and the Bessel string.

Furthermore, the results above on determined nonlattice cases confirm the conjecture, for these cases, that the authors formulate in [5, p.66] or [6, Section 3.7.1, p.111] through several numerical experiments and figures: 'Since there are no horizontal pieces in these graphs, we might conjecture that the real parts of their zeros are dense in $\left[D_{l}, D\right]$ ".

Finally, we also formulate a precise conjecture motivated by the numerical experiments performed on $R_{L_{n}}$ for $n \geq 2$.

\section{First properties of the functions $L_{n}(z)$}

As we observed above, the study of the complex dimensions of nonlattice strings is most naturally carried out in the more general setting of Dirichlet polynomials. The function $L_{n}(z)=1-2^{z}-\ldots-n^{z}, n \geq 2$, is clearly a Dirichlet polynomial. More generally, each entire function $L_{n}(z)$ belongs to the class of almost periodic functions [2], with spectrum formed by $\{\log 1, \log 2, \ldots, \log n\}$.

Furthermore, the functions $L_{n}(z)$ are related to the entire functions

$$
G_{n}(z):=1+2^{z}+\ldots+n^{z},
$$

which constitute a set of approximants of the Riemann zeta function on the halfplane Re $z<-1$, and they both satisfy similar properties. In fact, the direct relation between both functions is given by $G_{n}(z)+L_{n}(z)=2$ and $G_{n}^{\prime}(z)=-L_{n}^{\prime}(z)$. Hence, all zeros of the derivatives (of any order) of $G_{n}(z)$ and $L_{n}(z)$ are the same.

On the other hand, we can assure the existence of two real numbers $x_{1}$ and $x_{2}$ such that all zeros of $L_{n}(z)$ are in the vertical strip

$$
\left\{z \in \mathbb{C}: x_{1}<\operatorname{Re} z<x_{2}\right\}
$$

(see for example [8, Lemma 5]). The bounds above allow us to define an interval $\left[a_{L_{n}}, b_{L_{n}}\right]$, called critical interval, which contains the real projections of all zeros of each function $L_{n}(z)$, where

$$
a_{L_{n}}:=\inf \left\{\operatorname{Re} z: L_{n}(z)=0\right\}
$$


and

$$
b_{L_{n}}:=\sup \left\{\operatorname{Re} z: L_{n}(z)=0\right\} .
$$

It was also proved in [7] that the mean number of zeros of $G_{n}(z)$ is $\frac{\log n}{2 \pi}$. Now, according to [11, Theorem 3.2], this value is the same for the function $L_{n}(z)$. Besides, from [11, Theorem 5.2], the function $L_{n}(z)$ has less than $n$ zeros in a horizontal strip of width less than $\frac{2 \pi}{\log n}$.

Furthermore, according to [9, Theorem 3], there exist infinitely many $r_{n}$-rectangles $\left\{R_{n, T}\right\}$ in the critical strip of $L_{n}(z)$ such that the number of zeros, $N_{n}(T)$, of $L_{n}(z)$ inside each $R_{n, T}$ satisfies

$$
N_{n}(T)=\left[\frac{T \log n}{2 \pi}\right]+1,
$$

where $T$ is the height of $R_{n, T}$ and $[x]$ is used to denote the largest integer not exceeding the real number $x$.

Now, we will formulate some results on the extremes of the critical interval of $L_{n}(z)$ for each $n \geq 2$. Firstly, consider the function $L_{n}(x)=1-2^{x}-\ldots-n^{x}$ for real values of $x$. Since $L_{n}(x)$ is strictly decreasing, $L_{n}(x) \rightarrow-\infty$ when $x \rightarrow+\infty$ and $L_{n}(x) \rightarrow 1$ when $x \rightarrow-\infty$, the real function $L_{n}(x)$ has an unique zero. Hence, let $a_{L_{n}, 1}$ denote the unique real value that satisfies

$$
L_{n}\left(a_{L_{n}, 1}\right)=0 .
$$

Also, let $b_{L_{n}, 1}$ denote the value

$$
b_{L_{n}, 1}=\sup \left\{x \in \mathbb{R}: 1+2^{x}+\ldots+(n-1)^{x} \geq n^{x}\right\} .
$$

Proposition 2.1. Let $n$ be an integer with $n \geq 2$, then $a_{L_{n}}=a_{L_{n}, 1}$.

Proof. As $L_{n}\left(a_{L_{n}, 1}+i 0\right)=0$, from the definition of $a_{L_{n}}$, we obtain $a_{L_{n}, 1} \geq a_{L_{n}}$. On the other hand, it is the case that for any $x, y \in \mathbb{R}$

$\left|L_{n}(x+i y)\right|=\left|1-2^{x+i y}-\ldots-n^{x+i y}\right| \geq 1-\left|2^{x+i y}+\ldots+n^{x+i y}\right| \geq 1-2^{x}-\ldots-n^{x}$.

That is,

$$
\left|L_{n}(x+i y)\right| \geq L_{n}(x)
$$

Now, since $a_{L_{n}, 1}$ is the unique solution of $L_{n}(x)=0$ and the fact that $L_{n}(x)$ is strictly decreasing, we get

$$
\left|L_{n}(x+y i)\right|>0 \text { for any } x<a_{L_{n}, 1} .
$$

Hence $a_{L_{n}} \geq a_{L_{n}, 1}$ and thus $a_{L_{n}}=a_{L_{n}, 1}$.

Furthermore, we can provide an upper bound for the maximum of the critical interval of $L_{n}(z)$.

Proposition 2.2. Let $n$ be an integer number with $n \geq 2$, then $b_{L_{n}} \leq b_{L_{n}, 1}$.

Proof. For any $x, y \in \mathbb{R}$, we have

$\left|1-2^{x+i y}-\ldots-(n-1)^{x+i y}\right| \leq 1+\left|2^{x+i y}\right|+\ldots+\left|(n-1)^{x+i y}\right|=1+2^{x}+\ldots+(n-1)^{x}$.

Thus,

$\left|L_{n}(x+y i)\right| \geq n^{x}-\left|1-2^{x+i y}-\ldots-(n-1)^{x+i y}\right| \geq n^{x}-\left(1+2^{x}+\ldots+(n-1)^{x}\right)$. 
Consequently, from definition (2.4) of $b_{L_{n}, 1}$, we deduce that

$$
\left|L_{n}(x+y i)\right|>0 \text { for any } x>b_{L_{n}, 1} .
$$

Hence $b_{L_{n}} \leq b_{L_{n}, 1}$.

Next, consider the set $\Lambda$ formed by the weights of $L_{n}(z), \Lambda=\{\log j\}, j \geq 2$, then it is easy to prove that the set $\left\{\log p_{j}\right\}$, with $p_{j}$ the $j^{\text {th }}$ prime, is a basis of $\Lambda$ :

Remark 2.1. Let $n$ be an integer greater than 2 and $\left\{p_{1}, p_{2}, \ldots, p_{k_{n}}\right\}$ the set of all prime numbers less than or equal to $n$. Then the set

$$
\left\{\log p_{1}, \log p_{2}, \ldots, \log p_{k_{n}}\right\}
$$

is linearly independent over the rationals, i.e. any linear combination $n_{1} \log p_{1}+$ $n_{2} \log p_{2}+\ldots+n_{k_{n}} \log p_{k_{n}}=0$, with integers $n_{1}, n_{2}, \ldots, n_{k_{n}}$, implies $n_{1}=n_{2}=\ldots=$ $n_{k_{n}}=0$. Furthermore, there exist integers $\left\{l_{m j}: m=2, \ldots, n ; j=1,2, \ldots, k_{n}\right\}$ such that

$$
\log m=\sum_{j=1}^{k_{n}} l_{m j} \log p_{j}
$$

for each $m=2, \ldots, n$.

Thus, the following theorem allows us to give a first characterization of the sets $R_{L_{n}} \subset\left[a_{L_{n}}, b_{L_{n}}\right]$. It can be considered as an ad hoc version of [1, Theorem 3.1] to be directly applied to our functions $L_{n}(z)$. The proof of this result is analogous to that of $[3$, Theorem 1$]$.

Theorem 2.3. Fixed an entire $n>2$, consider $B=\left\{\log p_{j}\right\}_{j=1}^{k_{n}}$, with $p_{k_{n}}$ the largest prime less than or equal to $n$. For each $1 \leq m \leq n$, let $c_{m}$ be the unique vector with non-negative integer components satisfying $\log m=\left\langle c_{m}, \mathbf{b}\right\rangle$, where $\mathbf{b}=\left(\log p_{1}, \log p_{2}, \ldots, \log p_{k_{n}}\right)$ and $<\cdot, \cdot>$ denotes the standard inner product in $\mathbb{R}^{k_{n}}$. If we define the function

$$
\begin{aligned}
F_{L_{n}}: \mathbb{R} \times \mathbb{R}^{k_{n}} & \rightarrow \mathbb{C} \\
\left(t, x_{1}, \ldots, x_{k_{n}}\right) & \mapsto 1-\sum_{m=2}^{n} m^{t} e^{<c_{m},\left(x_{1}, \ldots, x_{k_{n}}\right)>i},
\end{aligned}
$$

then $t \in R_{L_{n}}$ if and only if there exists some vector $\mathbf{x} \in \mathbb{R}^{k_{n}}$ such that $F_{L_{n}}(t, \mathbf{x})=0$.

It is also important to highlight the following density result which can be uniquely applied to Dirichlet polynomials with real weights linearly independent over the rationals (see [10, Theorem 10]).

Theorem 2.4. Let $n \geq 2$. If $P(z)=1+\sum_{j=1}^{n} m_{j} e^{w_{j} z}$ is an exponential polynomial with positive real frequencies $w_{1}<\ldots<w_{n}$ linearly independent over the rationals and $m_{j} \in \mathbb{C}$ such that $\left|m_{j}\right|=1$ for all $j=1, \ldots, n$, then $R_{P}=\left[a_{P}, b_{P}\right]$, where $R_{P}:=\overline{\{\operatorname{Re} z: P(z)=0\}}$ and $a_{P}$ and $b_{P}$ are the extremes of its critical interval. 
3. The Real projections of the zeros of $L_{n}(z)$ For the CASES $n=2,3,4,5$

For the case $n=2$, it is clear that all zeros of the function $L_{2}(z)=1-2^{z}$ are located on the imaginary axis. Hence $R_{L_{2}}=\{0\}$.

For $n=3$, the minimum of the critical interval, namely $a_{L_{3}}$, can be computed by applying Proposition 2.1. Thus $a_{L_{3}} \approx-0.7878849109$. On the other hand, the maximum of the critical interval, namely $b_{L_{3}}$, by Proposition 2.2 , is bounded above by the solution of $1+2^{x}=3^{x}$, which is $x=1$. Thus $b_{L_{3}} \leq 1$. Moreover, since $F_{L_{3}}(1, \pi, 0)=1-2^{1} e^{i \pi}-3^{1} e^{i 0}=0$, where the function $F_{L_{3}}$ is defined in Theorem 2.3, then $1 \in R_{L_{3}}$ and subsequently $1 \leq b_{L_{3}}$. Thus $b_{L_{3}}=1$. Finally, according to Theorem 2.4 , the set $R_{L_{3}}$ entirely coincides with $\left[a_{L_{3}}, b_{L_{3}}\right]$, i.e. the set of dimensions of fractality of the Two-Three string of scaling ratios $\frac{1}{2}$ and $\frac{1}{3}$, associated with $L_{3}(-z)$, entirely coincides with the interval $\left[-b_{L_{3}},-a_{L_{3}}\right]$.

The case $n=4$ is the first which does not have all its weights linearly independent over the rationals. Thus we will use Theorem 2.3 and it will be necessary to consider

$$
\begin{aligned}
F_{L_{4}}: \mathbb{R} \times[0,2 \pi)^{2} & \rightarrow \mathbb{C} \\
\left(t, x_{1}, x_{2}\right) & \mapsto 1-2^{t} e^{x_{1} i}-3^{t} e^{x_{2} i}-4^{t} e^{2 x_{1} i}
\end{aligned}
$$

and

$$
\begin{aligned}
F_{L_{5}}: \mathbb{R} \times[0,2 \pi)^{3} & \rightarrow \mathbb{C} \\
\left(t, x_{1}, x_{2}, x_{3}\right) & \mapsto 1-2^{t} e^{x_{1} i}-3^{t} e^{x_{2} i}-4^{t} e^{2 x_{1} i}-5^{t} e^{x_{3} i}
\end{aligned}
$$

That is, $F_{L_{4}}$ and $F_{L_{5}}$ are the functions that appear in Theorem 2.3, adjusted to the functions $L_{4}(z)$ and $L_{5}(z)$ respectively.

Notice that, by using Proposition 2.1, we numerically find the values $a_{L_{4}} \approx$ -1.082131498 and $a_{L_{5}} \approx-1.234844354$. Moreover, $b_{L_{4}}=b_{L_{4}, 1}$ or, equivalently, the maximum of the critical interval of $L_{4}(z)$ is given by the solution of the equation $1+2^{x}+3^{x}=4^{x}$, which is approximately 1.7305074 . Indeed, firstly, since $b_{L_{4}, 1} \in R_{L_{4}}$ (because $F_{L_{4}}\left(b_{L_{4}, 1}, \pi, \pi\right)=0$ ), then $b_{L_{4}, 1} \leq b_{L_{4}}$. Secondly, according to Proposition 2.2 , it follows that $b_{L_{4}} \leq b_{L_{4}, 1}$ and hence $b_{L_{4}}=b_{L_{4}, 1}$. Finally, also by Proposition $2.2, b_{L_{5}}$ is bounded above by the solution of $1+2^{x}+3^{x}+4^{x}=5^{x}$, which is approximately 2.4260127 .

Then we prove the following result.

Theorem 3.1. The set $R_{L_{4}}$ entirely coincides with $\left[a_{L_{4}}, b_{L_{4}}\right]$.

Proof. Consider the function

$$
\begin{aligned}
g_{L_{4}}: \mathbb{R} \times[0,2 \pi) & \rightarrow \mathbb{C} \\
\left(t, x_{1}\right) & \mapsto 1-2^{t} e^{x_{1} i}-4^{t} e^{2 x_{1} i}
\end{aligned}
$$

and the distance function $d(\cdot, 0)$. Observe that, fixed $t \in \mathbb{R}$, the function $x_{1} \rightarrow$ $g_{L_{4}}\left(t, x_{1}\right)$ is continuous. Now, we show some inequalities that will be used to prove the theorem:

- For all real $t$, we have

$$
\begin{gathered}
d\left(g_{L_{4}}(t, 3 \pi / 2), 0\right)=\left|1+i 2^{t}+4^{t}\right|=\sqrt{\left(1+4^{t}\right)^{2}+4^{t}}= \\
\sqrt{4^{2 t}+3 \cdot 4^{t}+1}>3^{t} .
\end{gathered}
$$

That is,

$$
d\left(g_{L_{4}}(t, 3 \pi / 2), 0\right)-3^{t}>0 \forall t \in \mathbb{R}
$$


- Taking $x_{1}=\pi$, we have $d\left(g_{L_{4}}(t, \pi), 0\right)=\left|1+2^{t}-4^{t}\right|$. Consequently,

$$
d\left(g_{L_{4}}(t, \pi), 0\right)<3^{t}
$$

if and only if

$$
\left(1+2^{t}-4^{t}\right)^{2}<9^{t} .
$$

Now, since the equation $\left(1+2^{t}-4^{t}\right)^{2}-9^{t}=0$, which is equivalent to $\left(1+2^{t}-4^{t}-3^{t}\right) \cdot\left(1+2^{t}-4^{t}+3^{t}\right)=0$, has two real zeros, $t_{1}=0$ and $t_{2}=b_{L_{4}} \approx 1.7305074$, and the fact that $\left(1+2^{t}-4^{t}\right)^{2}-9^{t}<0$ if $t \in\left(t_{1}, t_{2}\right)$, we obtain

$$
d\left(g_{L_{4}}(t, \pi), 0\right)-3^{t}<0 \text { if } t \in\left(t_{1}, t_{2}\right) .
$$

- Now, taking $x_{1}=0$, we have $d\left(g_{L_{4}}(t, 0), 0\right)=\left|1-2^{t}-4^{t}\right|$. Consequently,

$$
d\left(g_{L_{4}}(t, 0), 0\right)-3^{t}<0
$$

if and only if

$$
\left(1-2^{t}-4^{t}\right)^{2}-9^{t}<0 .
$$

In this case, $\left(1-2^{t}-4^{t}\right)^{2}-9^{t}=\left(1-2^{t}-4^{t}-3^{t}\right) \cdot\left(1-2^{t}-4^{t}+3^{t}\right)$ vanishes in $t_{3}=a_{L_{4}} \approx-1.082131498$ and $t_{4}=0$. Therefore, since $\left(1-2^{t}-4^{t}\right)^{2}-9^{t}<0$ in $\left(t_{3}, t_{4}\right)$, it follows that

$$
d\left(g_{L_{4}}(t, 0), 0\right)-3^{t}<0 \text { if } t \in\left(t_{3}, t_{4}\right) .
$$

Finally, from (3.1), (3.2), (3.3) and by using the continuity of the distance function, for each $t \in\left(t_{3}, t_{2}\right)$, there exists $x_{1}^{t} \in[0,2 \pi)$ such that

$$
d\left(g_{L_{4}}\left(t, x_{1}^{t}\right), 0\right)-3^{t}=0,
$$

i.e.

and subsequently

$$
g_{L_{4}}\left(t, x_{1}^{t}\right)=3^{t} e^{i A_{t}} \text { for some } A_{t} \in[0,2 \pi)
$$

$$
1-2^{t} e^{x_{1}^{t} i}-3^{t} e^{i A_{t}}-4^{t} e^{2 x_{1}^{t} i}=0,
$$

or equivalently

$$
F_{L_{4}}\left(t, x_{1}^{t}, A_{t}\right)=0
$$

Now, by taking Theorem 2.3 into account, we assure the density of the projection of the zeros of $L_{4}(z)$ in the critical interval $\left[a_{L_{4}}, b_{L_{4}}\right]$, as we had stated above.

Consequently, we proved that the complex roots of the nongeneric nonlattice equation $L_{4}(-z)=0$, mentioned in [6, p.70], satisfy this important density property in all their critical interval $\left[-b_{L_{4}},-a_{L_{4}}\right]$.

In the same manner, we will prove the following result for the case $n=5$.

Theorem 3.2. The set $\left[a_{L_{5}},-0.851212\right) \cup(-0.563367,2.3118)$ is included in $R_{L_{5}}$.

Proof. Define

$$
\begin{aligned}
g_{L_{5}}: \mathbb{R} \times[0,2 \pi)^{2} & \rightarrow \mathbb{C} \\
\left(t, x_{1}, x_{2}\right) & \mapsto 1-2^{t} e^{x_{1} i}-3^{t} e^{x_{2} i}-4^{t} e^{2 x_{1} i} .
\end{aligned}
$$

By numerically solving the corresponding equations, we get:

i) $d\left(g_{L_{5}}\left(t, \frac{3 \pi}{2}, 0\right), 0\right)-5^{t}=\left|1+3^{t}+4^{t}+i 2^{t}\right|-5^{t}>0$ if $t \in\left[a_{5}^{L}, 2.133035\right)$;

ii) $d\left(g_{L_{5}}(t, 0,0), 0\right)-5^{t}=\left|1-2^{t}-3^{t}-4^{t}\right|-5^{t}>0$ if $t \in(-0.851212,2.3118)$;

iii) $d\left(g_{L_{5}}(t, 0,0), 0\right)-5^{t}=\left|1-2^{t}-3^{t}-4^{t}\right|-5^{t}<0$ if $t \in\left(a_{L_{5}},-0.851212\right)$; 


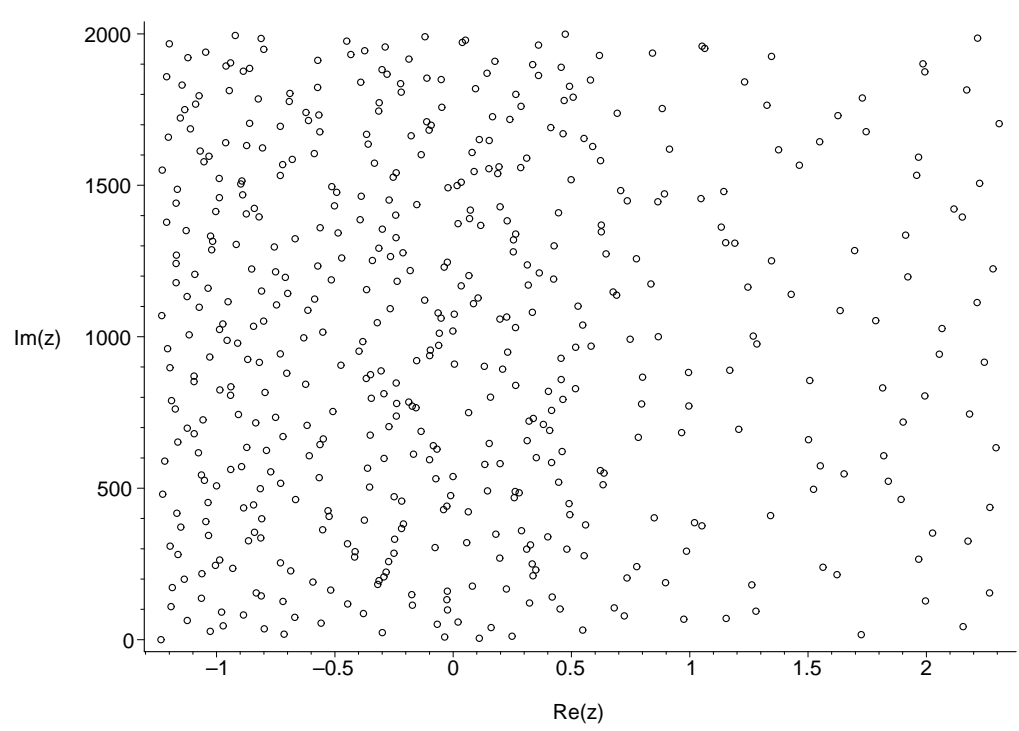

FIGURE 1. Zeros of $L_{5}(z)$ with imaginary part between 0 and 2000

iv) $d\left(g_{L_{5}}(t, 0, \pi), 0\right)-5^{t}=\left|1-2^{t}+3^{t}-4^{t}\right|-5^{t}<0$ if $t \in\left(-0.563367, b_{L_{5}}\right]$. Now, for each $t \in\left[a_{5}^{L},-0.851212\right) \cup(-0.563367,2.3118)$, by the continuity of the function $d(\cdot, 0)$ there exist $x_{1}^{t}, x_{2}^{t} \in[0,2 \pi)$ such that

$$
d\left(g_{L_{5}}\left(t, x_{1}^{t}, x_{2}^{t}\right), 0\right)-5^{t}=0 .
$$

That is,

$$
g_{L_{5}}\left(t, x_{1}^{t}, x_{2}^{t}\right)=5^{t} e^{i B_{t}} \text { for some } B_{t} \in[0,2 \pi)
$$

and subsequently

$$
1-2^{t} e^{x_{1}^{t} i}-3^{t} e^{x_{2}^{t} i}-4^{t} e^{2 x_{1}^{t} i}-5^{t} e^{i B_{t}}=0,
$$

or equivalently

$$
F_{L_{5}}\left(t, x_{1}^{t}, x_{2}^{t}, B_{t}\right)=0 .
$$

Finally, by taking Theorem 2.3 into account, the result follows.

\section{The Real projections of the zeros of $L_{n}(z)$ in the general case}

Unfortunately, the technique that we used for the cases $n=4$ and $n=5$ in order to compute $R_{L_{n}}=\overline{\left\{\operatorname{Re} z: L_{n}(z)=0\right\}}$ is heuristic and we can not generalize it for any integer $n \geq 2$.

In any case, from some numerical experiments and graphs plotted for the case $n=5$ and other values of $n>5$ (see for example Figures 1 and 2) we conjecture that $R_{L_{5}}=\left[a_{L_{5}}, b_{L_{5}}\right]$ and that this also occurs in the general case.

Conjecture 4.1. The set $R_{L_{n}}$ entirely coincides with $\left[a_{L_{n}}, b_{L_{n}}\right]$ for $n \geq 2$. 


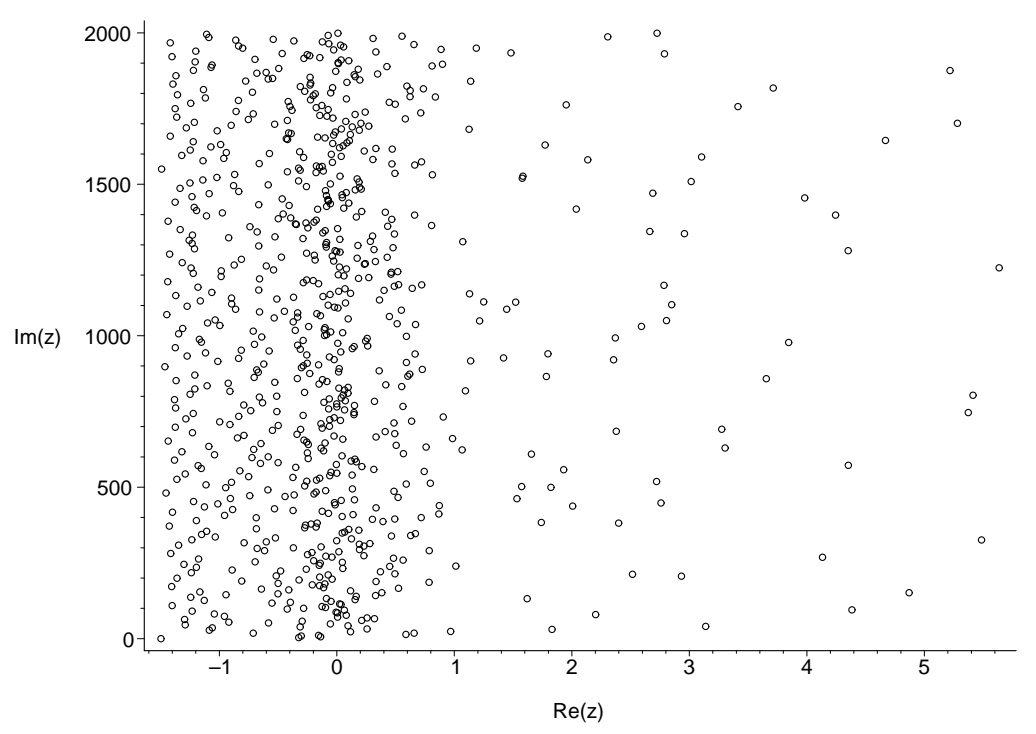

FigURE 2. Zeros of $L_{10}(z)$ with imaginary part between 0 and 2000

On the other hand, as we observed above, the upper bound $b_{L_{n}, 1}$ obtained in Proposition 2.2 for the maximum of the critical interval $\left[a_{L_{n}}, b_{L_{n}}\right]$ precisely coincides with this maximum $b_{L_{n}}$ for the cases $n=2,3,4$. We made numerical experiments in order to test this property (for example $b_{L_{10}, 1} \approx 5.89151$ and compare with Figure 2 ) and, originally, we also conjectured that:

"The value $b_{L_{n}, 1}$ defined in (2.4) coincides with $b_{L_{n}}$ for $n \geq 2$."

However, this conjecture fails for the case $n=5$. We will first prove that $L_{5}(z) \neq 0$ if $z$ is so that $\operatorname{Re} z=b_{L_{5}, 1}$. Observe that $b_{L_{5}, 1}$ is the solution to the equation $5^{x}=1+2^{x}+3^{x}+4^{x}$. If $L_{5}\left(z_{0}\right)=0$ for some $z_{0}$ such that Re $z_{0}=b_{L_{5}, 1}$, then

$$
5^{b_{5}, 1}=\left|5^{z_{0}}\right|=\left|1+2^{z_{0}}+3^{z_{0}}+4^{z_{0}}\right|=1+2^{\operatorname{Re} z_{0}}+3^{\operatorname{Re} z_{0}}+4^{\operatorname{Re} z_{0}},
$$

thus all the terms must point in the same direction to add up to the size of $5^{z_{0}}$. It follows that they must all be positive real. Hence $2^{z_{0}}$ and $4^{z_{0}}$ must be negative real numbers. But if $2^{z_{0}}<0$ then $4^{z_{0}}>0$, thus the terms $1,2^{z_{0}}, 3^{z_{0}}, 4^{z_{0}}$ add up to a size strictly smaller than $\left|5^{z_{0}}\right|$, which represents a contradiction. The idea for proving that the conjecture fails is the same: if $L_{5}\left(z_{0}\right)=0$ for $\operatorname{Re} z_{0}<b_{L_{5}, 1}$ but very close to $b_{L_{5}, 1}$, then all the terms must be close to positive real and, thus, $2^{z_{0}}$ and $4^{z_{0}}$ must be close to negative real numbers, which result in a contradiction. The conclusion is that the right-most complex dimensions must lie strictly to the left of $\operatorname{Re} z=b_{L_{5}, 1}$, by a fixed positive distance. A similar argument, applied to the same two terms $2^{z}$ and $4^{z}$, disproves the conjecture for every $n \geq 5$.

In any case, in this section we focus on general properties of $R_{L_{n}}$ in connection with Theorem 2.3.

Lemma 4.2. Let $n$ be an integer number greater than 2 and $z_{0}:=t_{0}+i y_{0}$ be a zero of $L_{n}(z)$. Then, by just taking $\mathbf{x}=y_{0} \mathbf{p}$, we have $F_{L_{n}}\left(t_{0}, \mathbf{x}\right)=0$, where $\mathbf{p}=\left(\log 2, \log 3, \ldots, \log p_{k_{n}}\right)$ and $p_{k_{n}}$ is the largest prime less than or equal to $n$. 
Proof. If $z_{0}:=t_{0}+i y_{0}$ is a zero of $L_{n}(z)$, then

$$
1-2^{t_{0}} 2^{i y_{0}}-3^{t_{0}} 3^{i y_{0}}-\ldots-n^{t_{0}} n^{i y_{0}}=0 .
$$

We are going to prove that there exist real numbers $x_{1}, x_{2}, \ldots, x_{k_{n}}$ such that

$$
F_{L_{n}}\left(t_{0}, x_{1}, x_{2}, \ldots, x_{k_{n}}\right)=0,
$$

where $k_{n}$ is the number of primes less than or equal to $n$. Indeed, take

$$
\begin{array}{rc}
x_{1}= & y_{0} \cdot \log 2 \\
x_{2}= & y_{0} \cdot \log 3 \\
x_{3}= & y_{0} \cdot \log 5 \\
\ldots & \cdots \\
x_{k_{n}-1}= & y_{0} \cdot \log p_{k_{n}-1} \\
x_{k_{n}}= & y_{0} \cdot \log p_{k_{n}},
\end{array}
$$

then

$$
F_{L_{n}}\left(t_{0}, x_{1}, x_{2}, \ldots, x_{k_{n}}\right)=1-2^{t_{0}} 2^{i y_{0}}-3^{t_{0}} 3^{i y_{0}}-\ldots-n^{t_{0}} n^{i y_{0}}=0 .
$$

Nextly, we take the following notation

$$
L_{n}^{*}(z):=L_{n}(z)+p_{k_{n}}^{z},
$$

with $p_{k_{n}}$ the largest prime less than or equal to $n$, and we will generalize the result above through the following lemma (observe that, in particular, a zero of $L_{n}(z)$ clearly satisfies the conditions of the following proposition).

Proposition 4.3. Fixed an integer $n>2$, let $t_{0} \in \mathbb{R}$ be such that there exists $y_{0} \in \mathbb{R}$ with $\left|L_{n}^{*}\left(t_{0}+i y_{0}\right)\right|=p_{k_{n}}^{t_{0}}$. Then, $t_{0} \in R_{L_{n}}$.

Proof. Let $g_{L_{n}}\left(t_{0}, x_{1}, x_{2}, \ldots, x_{k_{n}-1}\right)$ denote the function

$$
1-\sum_{m=2, m \neq p_{k_{n}}}^{n} m^{t} e^{<c_{m},\left(x_{1}, \ldots, x_{k_{n}-1}\right)>i} .
$$

If $t_{0}$ and $y_{0}$ satisfy $\left|L_{n}^{*}\left(t_{0}+i y_{0}\right)\right|=p_{k_{n}}^{t_{0}}$, by just taking

$$
\begin{array}{rc}
x_{1}= & y_{0} \cdot \log 2 \\
x_{2}= & y_{0} \cdot \log 3 \\
x_{3}= & y_{0} \cdot \log 5 \\
\ldots & \cdots \\
x_{k_{n}-1}= & y_{0} \cdot \log p_{k_{n}-1},
\end{array}
$$

one deduces

$$
\begin{aligned}
\left|g_{L_{n}}\left(t_{0}, x_{1}, x_{2}, \ldots, x_{k_{n}-1}\right)\right|= & \left|1-2^{t_{0}} 2^{i y_{0}}-3^{t_{0}} 3^{i y_{0}}-\ldots-n^{t_{0}} n^{i y_{0}}+p_{k_{n}}^{t_{0}} p_{k_{n}}^{i y_{0}}\right|= \\
& \left|L_{n}^{*}\left(t_{0}+i y_{0}\right)\right|=p_{k_{n}}^{t_{0}} .
\end{aligned}
$$

That is,

$$
g_{L_{n}}\left(t_{0}, x_{1}, x_{2}, \ldots, x_{k_{n}-1}\right)=p_{k_{n}}^{t_{0}} e^{i A_{t_{0}}} \text { for some } A_{t_{0}} \in[0,2 \pi) .
$$

Therefore, by taking $x_{k_{n}}=A_{t_{0}}$, we get

$$
g_{L_{n}}\left(t_{0}, x_{1}, x_{2}, \ldots, x_{k_{n}}\right)=0
$$


and, consequently, $t_{0} \in R_{L_{n}}$.

The converse result of the previous proposition is also true.

Proposition 4.4. Given an integer $n>2$ and $t_{0} \in R_{L_{n}}$, there exists $\lambda \in \mathbb{R}$ such that

$$
\left|L_{n}^{*}\left(t_{0}+i \lambda\right)\right|=p_{k_{n}}^{t_{0}} .
$$

Proof. If $t_{0} \in R_{L_{n}}$, there exists a sequence of zeros $\left(s_{m}=\sigma_{m}+i y_{m}\right)_{m=1,2, \ldots}$ of $L_{n}(z)$ such that

$$
t_{0}=\lim _{m \rightarrow \infty} \sigma_{m}
$$

By taking the definition of $L_{n}^{*}(z)$ into account, each zero $s_{m}$ of $L_{n}(z)$ satisfies $L_{n}^{*}\left(s_{m}\right)=p_{k_{n}}^{s_{m}}$. Thus, for each $m=1,2, \ldots$, we have

$$
\left|1-\sum_{j=2, j \neq p_{k_{n}}}^{n} j^{\sigma_{m}+i y_{m}}\right|=p_{k_{n}}^{\sigma_{m}} .
$$

Observe that the sequence $\left(e^{i y_{m}}\right)_{m=1,2, \ldots}$ is bounded, therefore there exists a subsequence $\left(e^{i y_{j} j}\right)_{j=1,2, \ldots}$ such that

$$
\lim _{j \rightarrow \infty} e^{i y_{m_{j}}}=e^{i \lambda} \text {, for some } \lambda \in[0,2 \pi) .
$$

Now, by considering the equality (4.1) for $m_{j}, j=1,2, \ldots$, and by taking $j \rightarrow \infty$, we have

or equivalently

$$
\left|1-\sum_{j=2, j \neq p_{k_{n}}}^{n} j^{t_{0}} e^{i \lambda \log j}\right|=p_{k_{n}}^{t_{0}},
$$

$$
\left|L_{n}^{*}\left(t_{0}+i \lambda\right)\right|=p_{k_{n}}^{t_{0}}
$$

Consequently, from Proposition 4.3 and Proposition 4.4, we have proved the following result that characterizes the points of the set $R_{L_{n}}$ through the level curves of $L_{n}^{*}$.

Theorem 4.5. A real number $t_{0}$ is an element of $R_{L_{n}}$ if and only if the level curve $\left|L_{n}^{*}(z)\right|=p_{k_{n}}^{t_{0}}$ meets the vertical line $x=t_{0}$.

Now, we will prove the following result which let us obtain some intervals of the set $R_{L_{n}}$ by studying the zeros of $L_{n}^{*}(z)$.

Corollary 4.6. Let $n \in \mathbb{N}, n \geq 4$, be a non-prime number and $z_{0}$ a zero of $L_{n}^{*}(z)$ such that $\operatorname{Re} z_{0} \geq 0$, then there exists a non-void interval $J$ containing $\operatorname{Re}\left(z_{0}\right)$ and so that $J \subset R_{L_{n}}$.

Proof. Let $z_{0}:=t_{0}+i y_{0}$ be a zero of $L_{n}^{*}(z)$, with $t_{0} \geq 0$. By continuity, determine a disk $D\left(z_{0}, r\right)=\left\{z \in \mathbb{C}:\left|z-z_{0}\right|<r\right\}$ such that

$$
\left|L_{n}^{*}(z)\right| \leq p_{k_{n}}^{t_{0}} \text { for any } z=t+i y \in D\left(z_{0}, r\right) .
$$

In particular, we have

$$
\left|L_{n}^{*}(t+i y)\right| \leq p_{k_{n}}^{t_{0}} \leq p_{k_{n}}^{t} \text { for any } t \geq t_{0} \text { and } t+i y \in D\left(z_{0}, r\right) .
$$


On the other hand, we have

$$
\left|L_{n}^{*}(t+i 0)\right|=\left|1-\sum_{j=2, j \neq p_{k_{n}}}^{n} j^{t}\right|=\left|s^{2}+\sum_{j=3, j \neq p_{k_{n}}}^{n} j^{t}\right|,
$$

where $s \in \mathbb{R}$ is so that $1-2^{t}=-s^{2}$ in the case $t \geq 0$. Therefore, since $p_{k_{n}} \neq n$, we have

$$
\left|L_{n}^{*}(t+i 0)\right| \geq p_{k_{n}}^{t} \text { for any } t \geq t_{0}
$$

Then, from (4.2) and (4.3), for each $t \geq t_{0}$ satisfying (4.2), the level curve $\left|L_{n}^{*}(z)\right|=$ $p_{k_{n}}^{t}$ meets the vertical line $x=t$. Consequently, according to Theorem 4.5 , there is an interval $J=\left[t_{0}, t_{0}+r\right)$ such that $J \subset R_{L_{n}}$. The proof is then complete.

Now, we will directly obtain some points that are in the set $R_{L_{n}}$.

Corollary 4.7. Let $n \geq 2$ be an integer and consider $D$ the solution set of the equation

$$
-1+2^{x}+\ldots+\left(p_{k_{n}}-1\right)^{x}-p_{k_{n}}^{x}+\left(p_{k_{n}}+1\right)^{x}+\ldots+n^{x}=0
$$

then $a_{L_{n}} \in R_{L_{n}}$ and $d \in R_{L_{n}}$ for each $d \in D$.

Proof. The number of zeros of the function

$$
h_{n}(x):=-1+2^{x}+\ldots+\left(p_{k_{n}}-1\right)^{x}-p_{k_{n}}^{x}+\left(p_{k_{n}}+1\right)^{x}+\ldots+n^{x}
$$

depends on the value of $n$. Indeed,

i) if $n$ is not a prime number, then the derivative of $h_{n}(x)$ is strictly increasing. Now, by using that $h_{n}(x) \rightarrow+\infty$ when $x \rightarrow+\infty$ and $h_{n}(x) \rightarrow-1$ when $x \rightarrow-\infty$, then $h_{n}(x)$ has an unique zero.

ii) if $n$ is a prime number, then $h_{n}(x) \rightarrow-\infty$ when $x \rightarrow+\infty$ and $h_{n}(x) \rightarrow-1$ when $x \rightarrow-\infty$. Moreover, $h_{n}^{\prime}(x)$ can take the value 0 and, thus, the function $h_{n}(x)$ is not necessarily monotone on $\mathbb{R}$ and the equation $h_{n}(x)=0$ does not necessarily have a unique solution.

Now, observe that $a_{L_{n}, 1}$ (the unique solution of $L_{n}(x)=0$ that coincides with $a_{L_{n}}$, by Proposition 2.1) and each point of the set $D$, the solution set of $h_{n}(x)=0$, satisfies $L_{n}^{*}(x)=p_{k_{n}}^{x}$ and $-L_{n}^{*}(x)=p_{k_{n}}^{x}$ respectively, i.e. $\left|L_{n}^{*}(x)\right|=p_{k_{n}}^{x}$. Then, by applying Theorem 4.5 , the result holds.

\section{Applications to Other nONLATtice STRINGS}

In this last section we will extend the results of Section 3, obtained for $R_{L_{n}}$, $n=2,3,4$, to the set of dimensions of fractality of other known nonlattice fractal strings.

Firstly, we will show some special fractal strings which can be identified to the generic nonlattice self-similar case, associated to Dirichlet polynomials with weights that are linearly independent over the rationals. This is the case of the Two-Three string, related to $R_{L_{3}}$, that was already studied above. Now, consider the following strings:

- The Two-Three-Five string [6, Example 3.48] is a self-similar string with one gap and three scaling ratios $r_{1}=\frac{1}{2}, r_{2}=\frac{1}{3}$ and $r_{3}=\frac{1}{5}$. Its complex dimensions can be calculated through the underlying Dirichlet polynomial

$$
P_{3}(z)=1-e^{z \log 2}-e^{z \log 3}-e^{z \log 5}
$$


of weights $\log 2, \log 3$ and $\log 5$ that, by Remark 2.1, are linearly independent over the rationals.

- The generic nonlattice case [6, Example 3.57] given by the Dirichlet polynomial

$$
P_{4}(z)=1-e^{z \log 2}-e^{z \log 3}-e^{z \log 5}-e^{z \log 7}
$$

of weights $\log 2, \log 3, \log 5$ and $\log 7$ that, by Remark 2.1, are also linearly independent over the rationals.

- The golden string [6, Example 3.46] is a self-similar string with a single gap and scaling factors $r_{1}=\frac{1}{2}$ and $r_{2}=\frac{1}{2^{\phi}}$, where $\phi$ is the golden ratio given by $\phi=\frac{1+\sqrt{5}}{2}$. Therefore, it is associated to

$$
G S(z)=1-e^{z \log 2}-e^{z \log 2^{\phi}} .
$$

Observe that $\log 2$ and $\log 2^{\phi}$ are linearly independent over $\mathbb{Q}$. Indeed, if $a$ and $b$ are rational numbers different from 0 , then

$$
a \log 2+b \log 2^{\phi}=a \log 2+b \log \left(2^{\frac{1}{2}} 2^{\frac{\sqrt{5}}{2}}\right)=\left[a+\frac{b}{2}+\frac{b \sqrt{5}}{2}\right] \log 2
$$

and, therefore, $a \log 2+b \log 2^{\phi}$ is equal to 0 if and only if $\frac{a}{b}=-\frac{1+\sqrt{5}}{2}$, which is not possible because the left term is rational and the right one is not rational. Thus $a=b=0$ and so $\log 2$ and $\log 2^{\phi}$ are linearly independent over $\mathbb{Q}$.

- The Bessel string [6, Example 3.47] is defined as the nongeneric nonlattice string of scaling ratios $\frac{1}{2}$ and $\frac{1}{2^{\alpha}}$, where $\alpha$ is the positive real number with continued fraction $[[1,2,3,4, \ldots]]$. So, $\alpha$ is not a rational number because its continued fraction representation is not finite. Hence we take

$$
\operatorname{Be}(z)=1-e^{z \log 2}-e^{z \alpha \log 2} .
$$

Observe that $\log 2$ and $\log 2^{\alpha}$ are also linearly independent over $\mathbb{Q}$. Indeed, if $a$ and $b$ are rational numbers different from 0 , then

$$
a \log 2+b \log 2^{\alpha}=a \log 2+b \alpha \log 2=(a+b \alpha) \log 2 .
$$

Hence, $a \log 2+b \log 2^{\alpha}$ is equal to 0 if and only if $\frac{a}{b}=-\alpha$, which is not possible because the left term is rational and the right one is not rational. Thus $a=b=0$ and so $\log 2$ and $\log 2^{\alpha}$ are linearly independent over $\mathbb{Q}$.

- The nonlattice fractal strings with two scaling ratios $r_{1}=\frac{1}{2}$ and $r_{2}=$ $2^{-1-\sqrt{2}}$ and with $r_{1}=\frac{1}{4}$ and $r_{2}=2^{-1-\sqrt{2}}$ with a single gap of length respectively $g_{1}=\frac{1}{2}-2^{-1-\sqrt{2}}$ and $g_{1}=\frac{1}{4}-2^{-1-\sqrt{2}}$ [6, Example 3.5]. They are associated to the Dirichlet polynomials $C(z)=1-2^{z}-2^{(1+\sqrt{2}) z}$ and $D(z)=1-4^{z}-2^{(1+\sqrt{2}) z}$.

From Theorem 2.4, the real projections of the zeros of $P_{3}(z), G S(z), B e(z), C(z)$ and $D(z)$ are dense in their corresponding critical intervals. Equivalently, the set of dimensions of fractality of the Two-Three-Five, the golden and the Bessel strings entirely coincides with its respective critical interval.

Nextly, we consider the golden+ string [6, Section 2.2.2], defined as the nongeneric nonlattice string of scaling ratios $\frac{1}{2}, \frac{1}{2^{\phi}}$ and $\frac{1}{2^{2}}$, where $\phi$ is the golden ratio. So, we take

$$
G S_{+}(z)=1-2^{z}-2^{\phi z}-4^{z}=1-e^{z \log 2}-e^{z \phi \log 2}-e^{2 z \log 2} .
$$


In this case, since the weights are not all linearly independent over the rationals, we will use Theorem 2.3 in order to find its set of dimensions of fractality. Thus we will follow the ideas used in Section 3 for the function $L_{4}(z)$. Let

$$
\begin{aligned}
F_{G S_{+}}: \mathbb{R} \times[0,2 \pi)^{2} & \rightarrow \mathbb{C} \\
\left(t, x_{1}, x_{2}\right) & \mapsto 1-2^{t} e^{x_{1} i}-2^{\phi t} e^{x_{2} i}-4^{t} e^{2 x_{1} i}
\end{aligned}
$$

be the function of Theorem 2.3 adjusted to the function $G S_{+}(z)$. Let $\left[a_{\left.G S_{+}, b_{G S_{+}}\right]}\right.$ be the critical strip associated to the Dirichlet polynomial $G S_{+}(z)$ and define the numbers

$$
a_{G S_{+}, 1}:=\inf \left\{x \in \mathbb{R}: 1 \leq 2^{x}+2^{\phi x}+2^{2 x}\right\}
$$

and

$$
b_{G S_{+}, 1}:=\sup \left\{x \in \mathbb{R}: 1+2^{x}+2^{\phi x} \geq 2^{2 x}\right\} .
$$

Observe that, by continuity, it follows that $1=2^{a_{G S_{+}, 1}}+2^{\phi a_{G S_{+}, 1}}+2^{2 a_{G S_{+}, 1}}$ and $1+2^{b_{G S_{+}, 1}}+2^{\phi b_{G S_{+}, 1}} \geq 2^{2 b_{G S_{+}, 1}}$.

Lemma 5.1. It is verified that $a_{G S_{+}}=a_{G S_{+}, 1}$ and $b_{G S_{+}}=b_{G S_{+}, 1}$.

Proof. Firstly, we will prove that $b_{G S_{+}} \leq b_{G S_{+}, 1}$. Indeed, if $z=x+i y$, with $x>b_{G S_{+}, 1}$, then

$$
2^{2 x}>1+2^{x}+2^{\phi x} \geq\left|1+2^{z}+2^{\phi z}\right| .
$$

Therefore, $G S_{+}(z) \neq 0$ for $x>b_{G S_{+}, 1}$ and the result follows. On the other hand, observe that $F_{G S_{+}}\left(b_{G S_{+}, 1}, \pi, \pi\right)=1+2^{b_{G S_{+}, 1}}+2^{b_{G S_{+}, 1} \phi}-2^{2 b_{G S_{+}, 1}}=0$. That is, $b_{G S_{+}, 1}$ is a point of the set formed by the closure of the real parts of the zeros of $G S_{+}(z)$ and thus $b_{G S_{+}, 1} \leq b_{G S_{+}}$. Subsequently, $b_{G S_{+}, 1}=b_{G S_{+}}$.

Now, we will prove that $a_{G S_{+}} \geq a_{G S_{+}, 1}$. If this is not so, $a_{G S_{+}}<a_{G S_{+}, 1}$, and noticing that

$$
a_{G S_{+}}:=\inf \left\{\operatorname{Re} z: G S_{+}(z)=0\right\},
$$

there exists a zero $v=t+i u$ of $G S_{+}(z)$ with $t<a_{G S_{+}, 1}$. Then, from the definition of $a_{G S_{+}, 1}$, we have

$$
1>2^{t}+2^{t \phi}+2^{2 t}
$$

Now, as $G S_{+}(v)=0$, we get

$$
2^{v}+2^{v \phi}+2^{2 v}=1
$$

and, by taking the modulus, it holds that

$$
1=\left|2^{v}+2^{v \phi}+2^{2 v}\right| \leq 2^{t}+2^{t \phi}+2^{2 t}
$$

which contradicts (5.1). Then $a_{G S_{+}} \geq a_{G S_{+}, 1}$, as claimed. Furthermore, since $G S_{+}\left(a_{G S_{+}, 1}+i 0\right)=0$, it is clear that $a_{G S_{+}} \leq a_{G S_{+}, 1}$ and the equality follows.

Numerically, the extremes of the critical interval are given by $a_{G S_{+}}=-1.074468745$ and $b_{G S_{+}}=1.780371302$ (also see Figure 3). We will demonstrate that the real parts of the complex dimensions of the Golden+ string $G S_{+}(z)$ are dense in its critical interval $\left[a_{G S_{+}}, b_{G S_{+}}\right]$.

Theorem 5.2. The set of dimensions of fractality of the Golden+ string coincides with $\left[-b_{G S_{+}},-a_{G S_{+}}\right]$. 


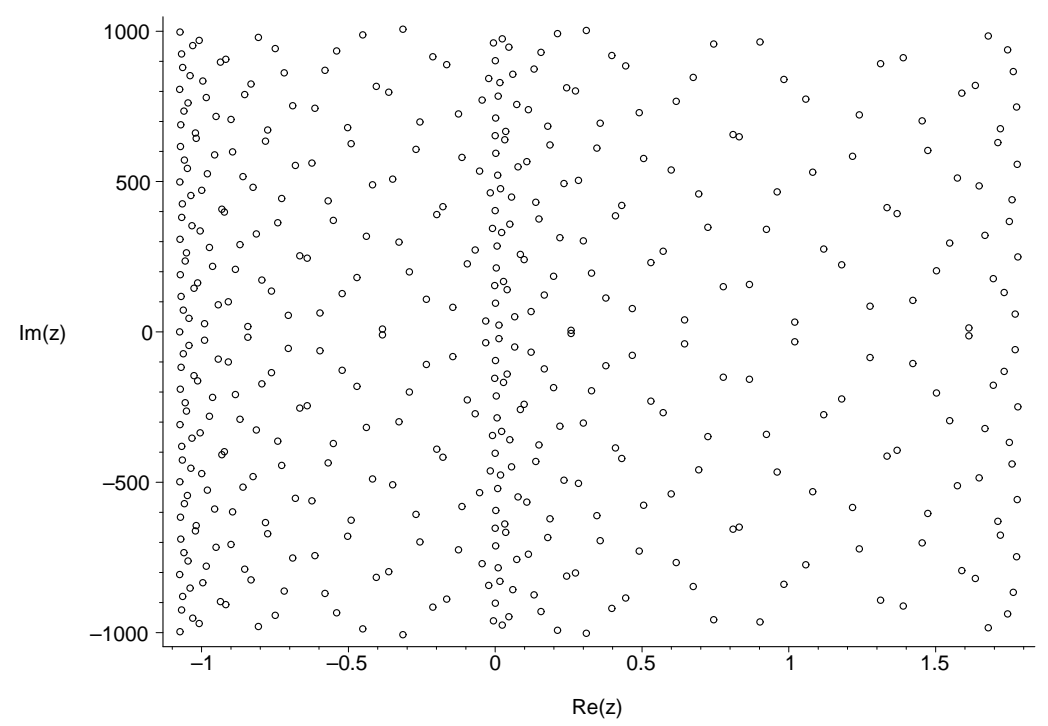

FiguRE 3. Zeros of $G S_{+}(z)$ with imaginary part between -1000 and 1000

Proof. Let

$$
\begin{aligned}
F_{G S_{+}}: \mathbb{R} \times[0,2 \pi)^{2} & \rightarrow \mathbb{C} \\
\left(t, x_{1}, x_{2}\right) & \mapsto 1-2^{t} e^{x_{1} i}-2^{\phi t} e^{x_{2} i}-4^{t} e^{2 x_{1} i}
\end{aligned}
$$

be the function that appears in Theorem 2.3, adjusted to the function $G S_{+}(z)$. We also consider the function

$$
\begin{aligned}
g_{G S_{+}}: \mathbb{R} \times[0,2 \pi) & \rightarrow \mathbb{C} \\
\left(t, x_{1}\right) & \mapsto 1-2^{t} e^{x_{1} i}-4^{t} e^{2 x_{1} i} .
\end{aligned}
$$

Observe the following inequalities:

- For all real $t$, it is satisfied

$$
\begin{gathered}
d\left(g_{G S_{+}}(t, 3 \pi / 2), 0\right)=\left|1+i 2^{t}+4^{t}\right|=\sqrt{\left(1+4^{t}\right)^{2}+4^{t}}= \\
\sqrt{4^{2 t}+3 \cdot 4^{t}+1}>2^{\phi t} .
\end{gathered}
$$

That is,

$$
d\left(g_{G S_{+}}(t, 3 \pi / 2), 0\right)-2^{\phi t}>0 \forall t \in \mathbb{R} .
$$

- Taking $x_{1}=\pi$, we have $d\left(g_{G S_{+}}(t, \pi), 0\right)=\left|1+2^{t}-4^{t}\right|$. So,

$$
d\left(g_{G S_{+}}(t, \pi), 0\right)-2^{\phi t}<0
$$

if and only if

$$
\left(1+2^{t}-4^{t}\right)^{2}-2^{2 \phi t}<0 .
$$

Now, since $\left(1+2^{t}-4^{t}\right)^{2}-2^{2 \phi t}=\left(1+2^{t}-4^{t}-2^{\phi t}\right) \cdot\left(1+2^{t}-2^{2 t}+2^{\phi t}\right)$ has two real zeros, $t_{1}=0$ and $t_{2}=b_{G S+}$, and the fact that $\left(1+2^{t}-4^{t}\right)^{2}-2^{2 \phi t}<0$ 
if $t \in\left(t_{1}, t_{2}\right)$, we obtain

$$
d\left(g_{G S_{+}}(t, \pi), 0\right)-2^{\phi t}<0 \text { if } t \in\left(t_{1}, t_{2}\right) .
$$

- Taking $x_{1}=0$, we have $d\left(g_{G S_{+}}(t, 0), 0\right)=\left|1-2^{t}-4^{t}\right|$. Hence,

$$
d\left(g_{G S_{+}}(t, 0), 0\right)-2^{\phi t}<0
$$

if and only if

$$
\left(1-2^{t}-4^{t}\right)^{2}-2^{2 \phi t}<0 .
$$

In this case, it follows that

$$
\left(1-2^{t}-4^{t}\right)^{2}-2^{2 \phi t}=\left(1-2^{t}-4^{t}-2^{\phi t}\right) \cdot\left(1-2^{t}-4^{t}+2^{\phi t}\right)
$$

vanishes in $t_{3}=a_{G S+}$ and $t_{4}=0$. Therefore, as $\left(1-2^{t}-4^{t}\right)^{2}-2^{2 \phi t}<0$ if $t \in\left(t_{3}, t_{4}\right)$, one deduces that

$$
d\left(g_{G S_{+}}(t, 0), 0\right)-2^{\phi t}<0 \text { if } t \in\left(t_{3}, t_{4}\right) .
$$

Finally, from (5.2), (5.3), (5.4) and by using the continuity of the distance function, for each $t \in\left(a_{G S+}, b_{G S+}\right)$, there exists $x_{1}^{t} \in[0,2 \pi)$ satisfying

$$
d\left(g_{G S_{+}}\left(t, x_{1}^{t}\right), 0\right)-2^{\phi t}=0 .
$$

That is,

$$
g_{G S_{+}}\left(t, x_{1}^{t}\right)=2^{\phi t} e^{i A_{t}} \text { for some } A_{t} \in[0,2 \pi)
$$

and consequently

$$
1-2^{t} e^{x_{1}^{t} i}-2^{\phi t} e^{i A_{t}}-4^{t} e^{2 x_{1}^{t} i}=0
$$

or equivalently

$$
F_{G S_{+}}\left(t, x_{1}^{t}, A_{t}\right)=0 .
$$

Now, by an argument similar to that of Theorem 2.3 adjusted to the function $G S_{+}(z)[1$, Theorem 3.1], we assure the density of the projection of the zeros of $G S_{+}(z)$ in $\left[a_{G S+}, b_{G S+}\right]$ and the result follows.

From several figures associated to the nongeneric nonlattice cases $L_{4}(-z)$ and $P_{4}(-z)$ and a variety of other nonlattice strings (the string associated to the Dirichlet polynomials $C(z)$ and $D(z)$, the Bessel string, the golden string, the Two-ThreeFive string and the golden+ string, among others), M. Lapidus and M. van Frankenhuysen conjecture (see $[6$, Section 3.7.1, p.111] or [5, p.66]) that "Since there are no horizontal pieces in these graphs, we might conjecture that the real parts of their zeros are dense in $\left[D_{l}, D\right]$ ". Therefore, note that in this paper we proved this conjecture for the mentioned cases, showing the density in their corresponding maximum connected intervals.

Acknowledgements. The authors wish to thank the referees for their helpful comments. We are especially grateful to one of the referees for suggesting the comments on the falseness of the conjecture, related to the right end-point of the critical interval, that we originally formulated. 


\section{REFERENCES}

[1] Avellar, C.E.; Hale, J.K.: On the zeros of exponential polynomials. Journal of Mathematical Analysis and Applications 73, 434-452 (1980).

[2] Bohr, H.: Almost Periodic Functions, Chelsea Publ. Comp., New York, 1947.

[3] Dubon, E.; Mora, G.; Sepulcre, J.M.; Ubeda J.I.; Vidal, T.: A note on the real projection of the zeros of partial sums of Riemann zeta function. Rev. R. Acad. Cienc. Exactas Fís. Nat. Ser. A Mat. RACSAM, DOI:10.1007/s13398-012-0094-2, 18 pages (2012).

[4] Lapidus, M.L., Pomerance, C.: The Riemann zeta-function and the one-dimensional WeylBerry conjecture for fractal drums. Proc. London Math. Soc. (3) 66, 41-69 (1993).

[5] Lapidus, M.L.; van Frankenhuysen, M.: Complex dimensions of self-similar fractal strings and Diophantine approximation. Experimental Mathematics 12, 1, 41-69 (2003).

[6] Lapidus, M.L.; van Frankenhuysen, M.: Fractal geometry, complex dimensions and zeta functions: Geometry and Spectra of Fractal Strings. Springer Monographs in Mathematics. Second edition. Springer, New York, 2013.

[7] Mora, G.; Sepulcre, J.M.: On the distribution of zeros of a sequence of entire functions approaching the Riemann zeta function. J. Math. Anal. Appl. 350, 409-415 (2009).

[8] Mora, G.; Sepulcre, J.M.: The critical strips of the sums $1+2^{z}+\ldots+n^{z}$, Abstract and Applied Analysis, vol. 2011, Article ID 909674, DOI:10.1155/2011/909674, 15 pages (2011).

[9] Mora, G.; Sepulcre, J.M.: Privileged regions in critical strips of non-lattice Dirichlet polynomials, Complex Analysis and Operator Theory, DOI: 10.1007/s11785-012-0248-4, 8 pages (2012).

[10] Mora, G.; Sepulcre, J.M.; Vidal, T.: On the existence of exponential polynomials with prefixed gaps, Bull. London Math. Soc., DOI: 10.1112/blms/bdt043, 16 pages (2013).

[11] Soprunova, E.: Exponential Gelfond-Khovanskii formula in dimension one. Proceedings of the American Mathematical Society, vol 136, n²1, 239-247 (2008).

Department of Mathematical Analysis, University of Alicante, 03080-Alicante, Spain E-mail address: eric.dubon@ua.es

Department of Mathematical Analysis, University of Alicante, 03080-Alicante, Spain E-mail address: JM.Sepulcre@ua.es 